\title{
A Testbed Platform to Demonstrate Spectrum Selection in Opportunistic Networks
}

\author{
Alessandro Raschellà, Anna Umbert, Jordi Pérez-Romero, Oriol Sallent \\ Department of Signal Theory and Communications \\ Universitat Politècnica de Catalunya (UPC) \\ Barcelona, Spain \\ e-mail: [alessandror,annau,jorperez,sallent]@tsc.upc.edu
}

\begin{abstract}
This paper presents a testbed platform to demonstrate and validate spectrum opportunity identification and spectrum selection functionalities in Opportunistic Networks (ONs). The hardware component of the testbed is based on reconfigurable devices able to transmit and receive data at different operating frequencies, which are dynamically configured. The software component has been developed to perform the creation and maintenance of ON radio links, including spectrum opportunity identification and selection decision making as well as all the necessary signaling to support the ON operation. Results presented in the paper validate the implementation conducted at the laboratory and illustrate the reconfigurability capabilities of the $\mathrm{ON}$ links under different conditions.
\end{abstract}

Keywords- Opportunistic Networks; Spectrum Selection; Spectrum Opportunity Identification; Testbed.

\section{INTRODUCTION}

It has been stated that the Internet has been successful because of its flexibility, its accessibility via different physical media, and for its simple support of many different types of applications and data types. Initially, wired access was dominant, while the set of applications was limited mainly to file-transfer, e-mail, media streaming and clientserver based web services/applications. In many positions on the Future Internet (FI), wireless access is expected to prevail, while at the same time there is growing interest for more application areas; thus, the FI is penetrating and covering almost every facet of our lives. For instance, increasingly modern information and communication services are built around social network concepts that require smart personal devices, and this makes it even more imperative to meet the need to offer appropriate connectivity everywhere where media or data flows need to be provided. Diversified applications/services can be accessed at any time of a day, can be requested from all types of locations/environments (e.g., home, public, work, urban, rural, etc.) or by all types of communication endpoints (e.g., machines, humans acting in different roles, namely in-work or private life), and can involve various information flows (voice, audio, data, images, video) and communication types (uni-cast, multicast, broadcast, peerto-peer). In contrast to today's Internet, for the FI, it can be safely assumed that the "best effort" delivery model will not hold. Certain applications, services and content will have to be delivered under Quality of Service (QoS) levels, or at least guaranteeing a certain Quality of Experience (QoE). Such hard requirements will set the networks under an enormous stress for resources (bandwidth, storage processing required) in both core and access parts. Traditionally, the need for more resources has been addressed through worst-case (peak-hour) based planning. This has lead to over-provisioning of resources in non-peak times. Keeping in mind that wireless resources are "expensive" (in the sense of "limited" or "scarce"), this over-provisioning will have to be tackled. In this respect, a range of solutions have been applied. For instance, many operators are aggressively adding $\mathrm{WiFi}$ access points and femtocell nodes to their network, in order to offload large portions of the traffic from the wide area networks of their infrastructure. However, as user behavior changes and user expectations increase, so do the resource requirements that are posed onto the communication networks. These continuously increasing requirements motivate the quest for further efficiency in resource provisioning.

Opportunistic Networks (ONs) are considered as an innovative solution to satisfy the demand for applications/services and respective resources, through increased efficiency in resource provisioning and utilization [1]. ONs are temporary, localised network segments created under certain circumstances. In this vision, ONs are always governed by the radio access network (RAN) operator (which provides the resources, the policies, the knowledge, etc.) so they can be considered as coordinated extensions of the infrastructure. ONs comprise both infrastructure nodes and infrastructure-less devices. The aim for a RAN operator to use ONs is to improve the performance of the infrastructure network, but also to provide a new span of localised or closed-group services. Further on, the introduction of cognitive techniques for the management of the ONs will lead to robustness and to capitalize the learning capabilities intrinsic to cognitive systems. ONs have been investigated to provide efficient solutions for a wide range of possible scenarios and use cases [2], such as: (1) "Opportunistic coverage extension", which describes a situation in which a device cannot connect to the operator's infrastructure, due to lack of coverage or a mismatch in the radio access technologies. The proposed solution includes an additional connected user that, by creating an opportunistic network, establishes a link between the initial device and the infrastructure, and acts as a data relay for this link. (2) "Opportunistic capacity extension", which depicts a 
situation in which a device cannot access the operator infrastructure due to the congestion of the available resources at the serving access node. The solution proposes the redirection of the access route through an ON that avoids the congested network segment. (3) "Infrastructure supported opportunistic ad-hoc networking", which considers the creation of a localised, infrastructureless $\mathrm{ON}$ among several devices for a specific purpose (peer-to-peer communications, home networking, etc.). Infrastructure governs the ON creation, benefits from the local traffic offloading and develops new opportunities for service provisioning. A common technical challenge in the different scenarios and $\mathrm{ON}$ use cases is to decide the proper spectrum to be used for the transmission of data and control flows in any communication link in accordance with the requirements for this link depending on the applications to be supported. This functionality is referred to as spectrum selection and it envisages a dynamic and flexible use of the available spectrum that ensures an efficient usage of this resource. The spectrum management process should be divided in two differentiated steps. First, the spectrum opportunity identification (SOI) will be in charge of finding out the set of possible frequency bands that are available for the link. Second, and based on the results of the previous step, the spectrum selection will decide the most adequate band for the communication. SOI and spectrum selection functionalities have been a topic of research in different studies. For instance, [3]-[5] proposed energy detection as a means to identify spectrum opportunities, while [6]-[10] present different algorithms and protocols for assigning spectrum in cognitive radio networks.

In this context, this paper describes the testbed implementation platform that has been developed for demonstrating and validating the spectrum selection functionality in ONs. It is built based on reconfigurable devices able to operate in different frequencies dynamically configured. This allows establishing and monitoring $\mathrm{ON}$ radio links, and reconfiguring them based on the changes in the current spectrum conditions. In this way, the testbed provides a practical insight for testing different algorithms in real environments, going beyond the purely theoretical analyses based on models and/or simulations.

The rest of the paper is structured as follows. In Section II, the ON life cycle and functional architecture for ON management are presented. Then Section III presents the algorithmic solutions for SOI and spectrum selection considered in the testbed and Section IV provides the testbed implementation. Section V presents some results and Section VI summarises the conclusions and next steps.

\section{OPPORTUNISTIC NETWORKS: LIFE CYCLE AND FUNCTIONAL ARCHITECTURE}

The life cycle of an ON comprises the following phases: (1) Suitability determination, where the convenience of setting up a new $\mathrm{ON}$ is assessed according to the triggering situation, previous knowledge, policies, profiles, etc., (2) Creation, which includes the selection of the optimal, feasible configuration for the new ON (selection of the participant nodes, the spectrum and the routing pattern), (3) Maintenance, which involves monitoring and controlling the QoS of the data flows involved in the ON and performing the appropriate corrective actions when needed, and (4) Termination, when the motivations for the creation of the $\mathrm{ON}$ disappear or the $\mathrm{ON}$ can no longer provide the required QoS and, therefore, mechanisms should be provided to handle handovers and to keep applications alive if possible.

Spectrum selection is involved in all the management stages in the ON life cycle. During suitability determination, which is the result of a rough feasibility analysis in order to keep complexity moderate, there is the need to introduce mechanisms leading to the identification of spectrum opportunities that ensure that the resulting interference conditions in the possible future $\mathrm{ON}$ will result acceptable. The suitability stage will provide one or several possible configurations for an $\mathrm{ON}$, whose feasibility and potential gains have been roughly estimated. Then, during the creation a detailed analysis (thus probably requiring additional context awareness and/or more accurate estimations related to diverse aspects of the radio environment) will be conducted and the spectrum to be assigned will be decided.

ON reconfiguration capabilities will provide the necessary adaptability to changing conditions. This stage comprises monitoring (i.e., dynamically acquire all the relevant information that may influence decision making processes around the $\mathrm{ON}$ such as relevant changes in the radio spectrum occupancy/interference conditions) and reconfiguration decisions. Reconfiguration decisions will be supported by other functionalities like discovery procedures for the identification of new nodes, identification of spectrum opportunities, etc. Based on the functional architecture (FA) proposed in [11] by the European Telecommunications Standardization Institute (ETSI) for Reconfigurable Radio Systems (RRSs) an extension was proposed in [12] to deal with ON management. In particular, the considered FA extends the ETSI's approach by adding two new cognitive management entities, to achieve close cooperation of the infrastructure and the ONs. These entities are the "Cognitive management Systems for Coordinating the Infrastructure" (CSCI) and the "Cognitive systems for Managing the Opportunistic Network" (CMON). Figure 1 provides a high level representation of the main functional blocks of the considered FA. The CSCI is mainly responsible for the activities before an $\mathrm{ON}$ is created. It is the functional entity in charge of acquiring and processing the context to carry out the ON suitability determination phase that detects the situations where an ON may be useful and determines whether or not right conditions are in place for creating an ON. The CSCI delegates the actual creation, maintenance and termination of a given $\mathrm{ON}$ to the associated CMON functional entity and it is located in both the operators' infrastructure side (namely "CSCI-N") and the terminal side (namely "CSCI-T"). The CMON is controlling the life cycle of the $\mathrm{ON}$ from creation to termination. This includes the execution of the creation procedures to enforce the design obtained from the CSCI, the supervision of the $\mathrm{ON}$ during maintenance phase and as well as the termination procedures. 


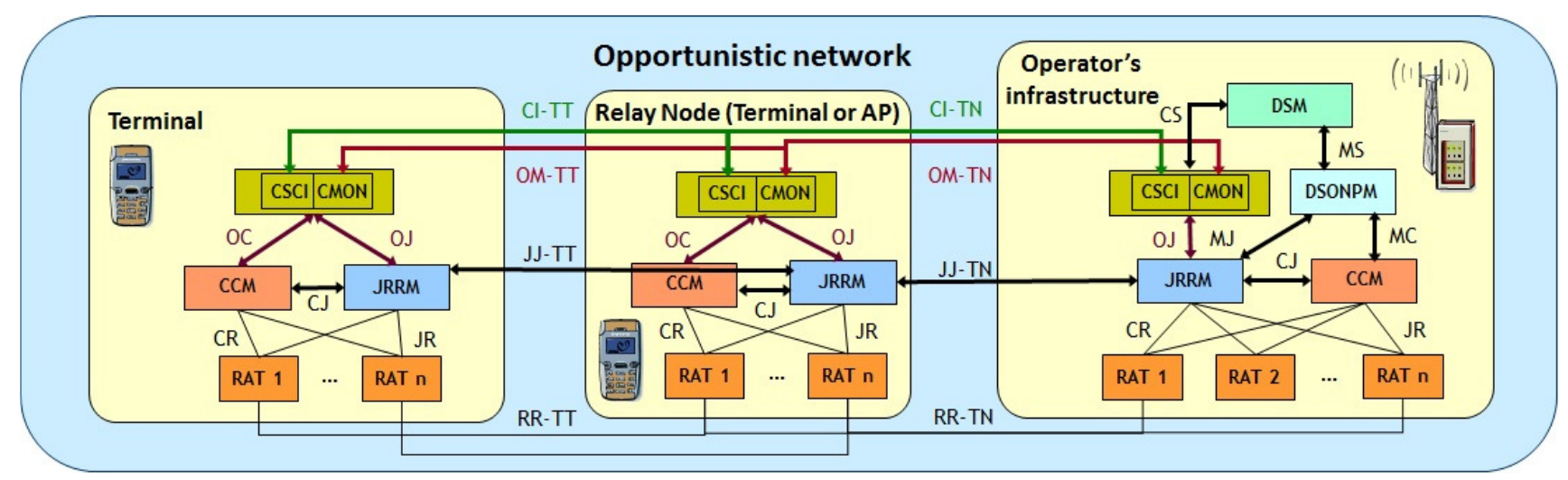

Figure 1. Considered FA, as an extension of ETSI RRS

Furthermore, the CMON is responsible for the coordination of nodes in the ON. The CMON is also located in both the operators' infrastructure (namely "CMON-N") and the terminal side (namely "CMON-T"). In general, the CMON in the operators' infrastructure involves context awareness, policy acquisition and profile management which provide the input for the decision making mechanism. In the terminal side, the CMON provides functionality for the context awareness, the policy acquisition as defined by the operator and the profile management. The cognition relies on the fact that the knowledge management functional entity interacts with the previously mentioned entities in order to make better decisions in the future, according to the learned results.

While in general the decision making related to $\mathrm{ON}$ management can be centralized, decentralized or mixed, this paper considers a fully centralized architecture, where ON management features are attributed to the infrastructure side. Figure 2 depicts an example and simplified view of such management entity at the infrastructure.

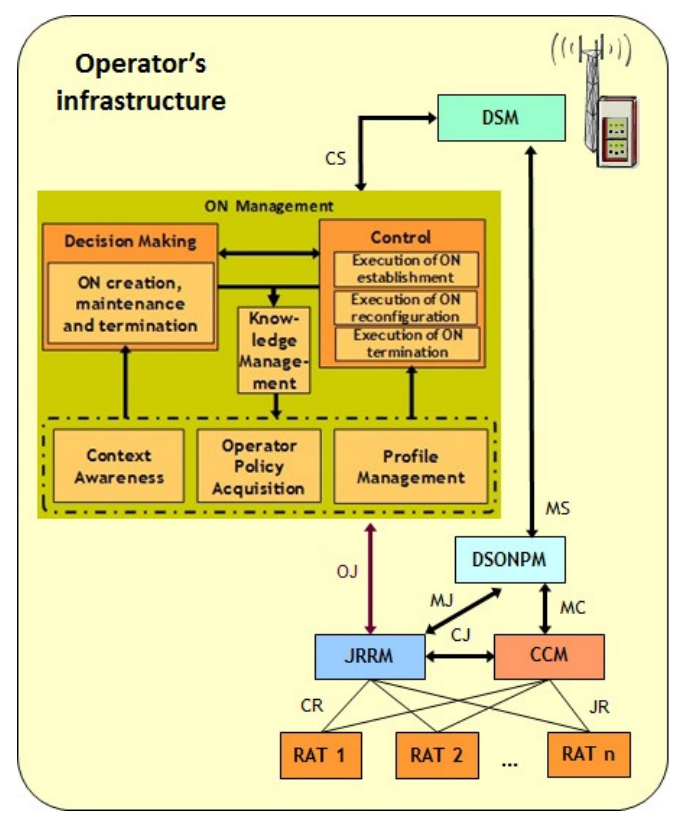

Figure 2. ON management at the infrastructure side
In Figure 2 the following elements are highlighted: (1) the decision-making processes associated to the different ON stages, (2) the control mechanisms that will lead to execute the decisions taken, (3) the knowledge management module to exploit cognitive features, (4) the context awareness to provide the necessary inputs about the radio environment conditions to the decision making algorithms, and (5) the Dynamic Spectrum Management (DSM) that provides the spectrum availability conditions and related constraints to guide the spectrum selection decision making.

\section{SPECTRUM SELECTION: ALGORITHMIC SOLUTIONS}

From an algorithmic perspective, the problem considered in the testbed presented in this paper is the selection of the spectrum to be assigned to a set of ON radio links between a pair of terminals and/or infrastructure nodes. The purpose of each radio link is to support a given application with certain bit rate requirements. The spectrum selection is carried out in the decision making entity and it is supported by the SOI residing in the DSM module.

\section{A. Spectrum Opportunity Identification algorithm}

The SOI algorithm executes two different procedures: the measurement procedure and the spectrum block formation.

In the measurement procedure, the total analysed band is subdivided into $N$ smaller portions of equal band $\Delta f$. The measurement algorithm performs an energy detection sensing (during a period of time $\Delta t$ ) for each $\Delta f$ portion until measuring the total band, starting from frequency F_min_band. This measurement is repeated Num_Meas times. Then, based on the multiple measurements carried out, the spectrum opportunity index is obtained for each portion, defined as the fraction of measurements in which this portion has been detected as available. The power threshold to decide if a portion is free is set based on [13].

In the spectrum block formation procedure, the consecutive spectrum blocks with spectrum opportunity index above a certain threshold are grouped in blocks. Each block is constituted by a maximum of $P_{\max }$ portions. For each block, the algorithm returns the 2-tuple $S B_{k}=\left\{f_{k}, B W_{k}\right\}$ where $f_{k}$ is the central frequency of the block and $B W_{k}$ the bandwidth. 


\section{B. Spectrum selection algorithm}

The spectrum selection algorithm uses as input the set of available spectrum pools resulting from the SOI, together with the characteristics of each pool in terms of available bit rate based on radio considerations. The algorithm output is the list of spectrum assignments to each of the existing ON radio links. The algorithm presented in [14] is considered for the implementation in the testbed. It makes use of the fittingness factor concept as a metric between 0 and 1 that capture how suitable a specific spectrum pool is for a specific radio link. The algorithm is based on estimating the fittingness factor for each link and available spectrum block based on a knowledge database that is maintained with different fittingness factor statistics. These statistics include the probabilities that the fittingness factor is kept to either high or low values as well as its time variability. The reader is referred to [14] for details.

\section{TESTBED IMPLEMENTATION}

In this section, the implemented testbed platform is described and details on the hardware and software components, the testbed architecture and the implemented signaling are provided.

\section{A. Hardware component: basic building block}

The testbed demonstrator is made of several nodes implemented through Universal Software Radio Peripheral (USRP) boards controlled by a laptop. Each USRP integrated board incorporates $\mathrm{AD} / \mathrm{DA}$ Converters (ADCs/DACs), a Radio Frequency (RF) front end, and a Field Programmable Gate Array (FPGA) which executes some pre-processing of the input signal [15]. A typical setup of the USRP board consists of one mother board and up to four daughter boards. On the mother board, there are four slots, where up to $2 \mathrm{RX}$ and 2 TX daughter boards can be plugged in. The daughter boards are used to hold the RF receiver and the RF transmitter. There are 4 high-speed 12-bit ADCs and 4 highspeed 14-bit DACs. All the ADCs and DACs are connected to the FPGA that performs high bandwidth math procedures such as filtering, interpolation and decimation. The DACs clock frequency is $128 \mathrm{Msample/s}$, while ADCs work at 64 Msample/s to digitize the received signal. A USB controller sends the digital signal samples to a PC in I/Q complex data format (4 bytes per complex sample), resulting in a maximum rate of $8 \mathrm{Msample/s}$. Consequently, the FPGA has to perform filtering and digital down-conversion (decimation) to adapt the incoming data rate to the USB 2.0 and PC computing capabilities. The maximum RF bandwidth that can be handled is thus $8 \mathrm{MHz}$.

There exist different kinds of daughter boards that allow a very high USRP reconfigurability and working at several frequency bands. The daughter boards integrated in the USRP motherboard of this testbed are XCVR2450 Transceivers. They work in the frequency ranges $2.4-2.5$ $\mathrm{GHz}$ and $4.9-5.9 \mathrm{GHz}$.

\section{B. Software Component}

Identification of spectrum opportunities is performed by both a hardware platform (i.e., USRP) and a software component implemented with GNU Radio toolkit. GNU Radio is a free and open source software for learning about, building and deploying software radios [16]. It provides a library of signal processing blocks and the glue to tie it all together. In GNU Radio, the programmer builds a radio by creating a graph where the vertices are signal processing blocks and the edges represent the data flow between them. All the signal processing blocks are written in $\mathrm{C}++$ and Python is used to create a network or graphs and glue these blocks together. Simplified Wrapper and Interface Generator (SWIG) is an open source package used by GNU Radio as glue such that the $\mathrm{C}++$ classes can be used from Python. SWIG has the ability to convert the $\mathrm{C}++$ classes into Python compatible ones. As a result, the whole GNU Radio framework is capable of putting together and exploiting the benefits of both $\mathrm{C}++$ and Python. GNU Radio has been used to develop the modules that implement the algorithms described in Section III and to enable the data and control communication between USRP transceivers.

\section{Testbed architecture}

The objective of this testbed is to show the behaviour of the SOI and spectrum selection procedures in an ON. For that purpose, a scenario is considered where two devices need to communicate through an ON link controlled by the infrastructure. Both SOI and spectrum selection functionalities reside in the infrastructure node.

In terms of physical implementation (see Fig. 3), USRP\#1 implements the infrastructure and the associated spectrum identification and selection functionalities, while USRP\#2 and USRP\#3 are the terminals exchanging data through the ON link.

In order to illustrate how the cognitive management system is able to detect and react to time-varying QoS conditions over the different spectrum blocks, a controlled interference source is also included. It is implemented as USRP\#4, whose operating frequency at each time can be defined as part of the testbed configuration set-up. Moreover, the periods in which the interference source is active or inactive are also controlled following specific random patterns whose statistic can also be configured. A single RAT using GMSK modulation at $256 \mathrm{Kbit} / \mathrm{s}$ is considered and, therefore, the JRRM module in ETSI RRS is omitted in the implemented testbed. ISM $2.4 \mathrm{GHz}$ band is used for the demonstration.

\section{Signalling procedures}

Since the target of the demonstration is the SOI and spectrum selection, the demonstration implements only the ON creation and ON maintenance stages of the ON life cycle. It is assumed that the decision to create an ON among the two devices has been previously made in the ON suitability phase.

The cognitive control channel signaling is implemented with the Control Channel for the Cooperation of the Cognitive Management System (C4MS) protocol using the implementation option based on IEEE 802.21 "MediaIndependent Handover (MIH) Services" [18]. 


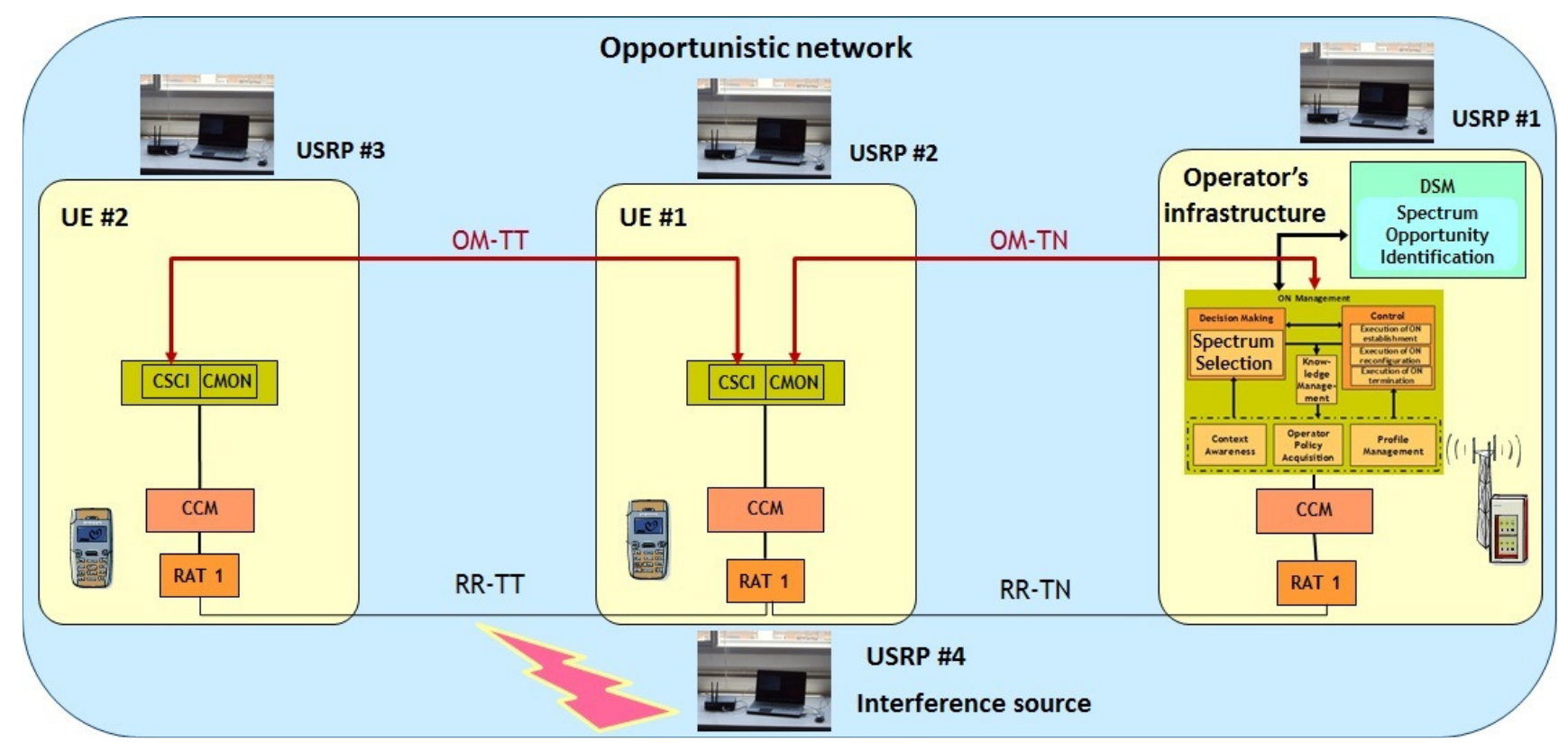

Figure 3. Scenario considered in the demonstration and corresponding implementation by means of USRP

For illustrative purposes, the implemented procedure for the ON creation is shown in Fig. 4. The steps of the procedure are the following:

1. A MIH_C4MS_ONN.request message is sent from UE\#1 to the infrastructure to start the ON-Negotiation $(\mathrm{ONN})$ procedure intended to obtain a valid configuration of the radio link. The message indicates the terminals involved and the QoS requirements that the link is expected to support, in terms of required bit rate.

2. The infrastructure sends a MIH_C4MS_ONN.request to UE\#2 informing it about the intention to establish a direct radio link with UE\#1 and allowing it to join the negotiation process for the derivation of the radio link configuration.

3. UE\#2 replies to the infrastructure with a MIH_C4MS_ONN.response message, notifying its acceptance for the establishment of the link.

4. The ON management entity in the infrastructure inquires the DSM entity to determine spectrum availability for the link. The SOI algorithm is executed.

5. DSM reply provides the available spectrum blocks, and the spectrum selection algorithm is executed to decide the spectrum block to be allocated to the link.

6 . The proposed ON configuration with the selected spectrum is transferred to UE\#1 by issuing a MIH_C4MS_ONN.response message.

7. To start the ON Creation (ONC), UE\#1 sends a MIH_C4MS_ONC.request to the infrastructure with the final ON configuration.

8. Infrastructure sends another MIH_C4MS_ONC.request towards UE\#2 with the final ON configuration.

9. UE\#2 replies with a MIH_C4MS_ONC.response message with a successful result-code indicating that the terminal is ready to establish the link.

10. Infrastructure concludes the $\mathrm{ON}$ creation procedure by sending a MIH_C4MS_ONC.response message to UE\#1.

11. The link establishment takes place at this point.

12. Finally, the creation of the $\mathrm{ON}$ is notified to the infrastructure from UE\#1 by sending a MIH_C4MS_ONSN.indication message.

A similar procedure is also implemented for the $\mathrm{ON}$ modification in case that degradation in the communication is perceived by one of the UEs. In this case, the procedure eventually triggers a new execution of the spectrum selection algorithm to modify the spectrum allocated to the link.

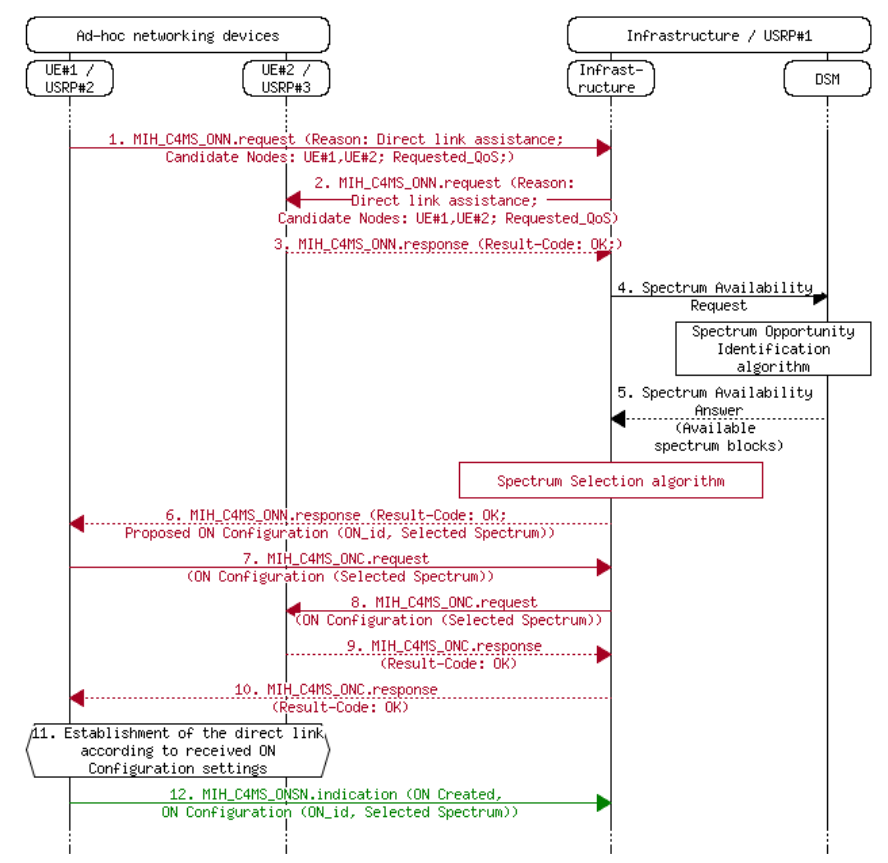

Figure 4. Implemented message exchange for the $\mathrm{ON}$ creation 


\section{VALIDATION RESULTS}

In order to illustrate the testbed operation, some validation and performance results of SOI and spectrum selection functionalities are presented in the following.

\section{A. Spectrum opportunity identification}

To test the SOI algorithm, the measurement procedure considered the total band from $2.4 \mathrm{GHz}$ to $2.5 \mathrm{GHz}$ subdivided in 1000 portions of $100 \mathrm{kHz}$. Energy detection sensing was performed for each portion during $100 \mathrm{~ms}$. The threshold to detect that a portion is available is set using the following procedure: (i) the USRP antenna was replaced with a matched load (i.e., a $50 \mathrm{ohm}$ resistor); (ii) the Cumulative Distribution Function (CDF) of the thermal noise was calculated; (iii) a threshold between thermal noise and signal energy was selected considering a false alarm probability equal to $1 \%$. In the considered test scenario, two WiFi access points transmitting at $2.412 \mathrm{GHz}$ and $2.432 \mathrm{GHz}$ were present.

Figure 5 presents the obtained spectrum opportunity indexes for all the 1000 portions of $100 \mathrm{kHz}$ averaged during a 10 minutes period. It can be observed that the spectrum portions in the ISM channel occupied by the WiFi access points have a spectrum opportunity index equal to $0 \%$. In turn, there are three groups of consecutive $100 \mathrm{kHz}$ blocks with a high opportunistic index value (i.e. greater than $80 \%$ ). As a result, the spectrum blocks provided by the algorithm are those indicated in Table I, considering that the maximum number of portions of a block has been set to $P_{\max }=290$. Correspondingly, the available set of portions between 2442 to $2500 \mathrm{MHz}$ with a total of $58 \mathrm{MHz}$ has been split into 2 blocks.

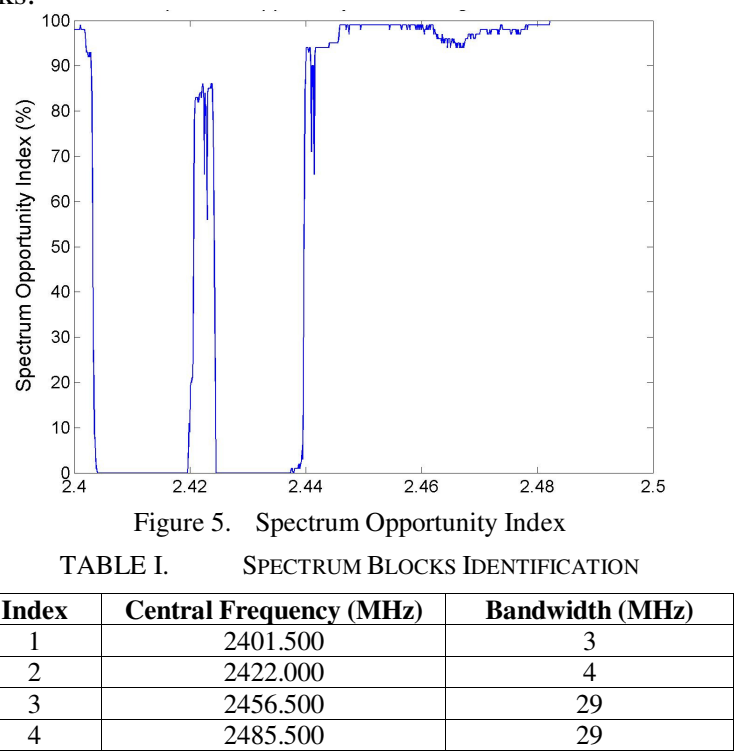

B. Adaptation of spectrum selection to interference variations

The aim of this subsection is to illustrate how the result of the SOI is used to perform the spectrum selection functionality. In the scenario illustrated in Fig. 3, the terminals (i.e., USRP\#2 and USRP\#3) will constitute an ON.
Following the procedure in Fig. 4, the allocated spectrum block will be decided by the infrastructure during the ONN procedure based on the SOI executed by USRP\#1 in the ISM $2.4 \mathrm{GHz}$ band. The identification procedure is the same explained in the previous sub-section, but now averaging the measurements during a period of $10 \mathrm{~s}$ and with $P_{\max }=50$. Firstly, USRP\#2 has sent the ONN.request message to USRP\#1. After receiving the ONN.request message from USRP\#2 and contacting USRP\#3 to confirm its availability to create the ON, USRP\#1 executes the SOI algorithm. Table II illustrates the result of the SOI at this stage. In this experiment USRP\#1 chooses the spectrum block \#6 centered at $2.447 \mathrm{GHz}$ for the operation of the ON between USRP\#2 and USRP\#3. This information is communicated to USRP\#2 in the ONN.response message. This triggers the ONC.request message to finalise the ON creation. After the $\mathrm{ON}$ has been created, the transmission of data between USRP\#2 and USRP\#3 starts on this specific spectrum block. The characteristics of data transmission for the communication between terminals are given in Table III.

TABLE II. SPECTRUM OPPORTUNITY IDENTIFICATION RESULT

\begin{tabular}{|c|c|c|}
\hline Index & Central Frequency (MHz) & Bandwidth (MHz) \\
\hline 1 & 2422 & 5 \\
\hline 2 & 2427 & 5 \\
\hline 3 & 2432 & 5 \\
\hline 4 & 2437 & 5 \\
5 & 2442 & 5 \\
\hline 6 & 2447 & 5 \\
\hline 7 & 2452 & 5 \\
\hline
\end{tabular}

At this stage, ON is in maintenance mode. USRP\#2 periodically monitors the efficiency in the data transmission as the ratio between successfully transmitted data packets and total number of transmitted data packets including retransmissions. This is computed based on the received acknowledgements for each packet.

TABLE III. EXPERIMENT ASSUMPTIONS

\begin{tabular}{|c|c|}
\hline Parameter & Bandwidth (MHz) \\
\hline Modulation & GMSK \\
\hline Data Rate & $256 \mathrm{kbps}$ \\
\hline Packet Size & $1500 \mathrm{byte}$ \\
Minimum Efficiency threshold & $80 \%$ \\
\hline Experiment Time & 25 minutes \\
\hline
\end{tabular}

In order to illustrate the capabilities of the cognitive management system to react to time-varying interference conditions and to adapt the selected spectrum block to provide good QoS for the ON communication, the interference source in the testbed platform (i.e., USRP\#4) is configured to transmit at different frequencies during the experiment (see Fig. 6). In this particular realization, after roughly 8 minutes running the testbed, USRP\#4 starts transmitting on the same frequency band used for ON data transmission. As a result, degradation in the communication is observed, as seen in Fig. 7 that depicts the evolution of the efficiency in the ON link communication together with the central frequencies of the spectrum blocks assigned to the ON. When the efficiency is below the threshold of $80 \%$, USRP\#2 triggers the ON-Modification (ONM) procedure, requesting for a new spectrum block where data 
communication can be continued with improved QoS. USRP\#2 sends an ONN.request message to the infrastructure starting the $\mathrm{ON}$ reconfiguration procedure. After executing again the SOI algorithm, the spectrum selection functionality decides that ON will continue operation through the spectrum block centered at $2.442 \mathrm{GHz}$. This is notified to USRP\#2 in the ONN.response message. After receiving this response, USRP\#2 sends the ONM.request to USRP\#3 to reconfigure the link in the new spectrum block. Then, the transmission between the terminals continues, reaching again high efficiency levels. The same process is illustrated twice during the rest of the demonstration time.

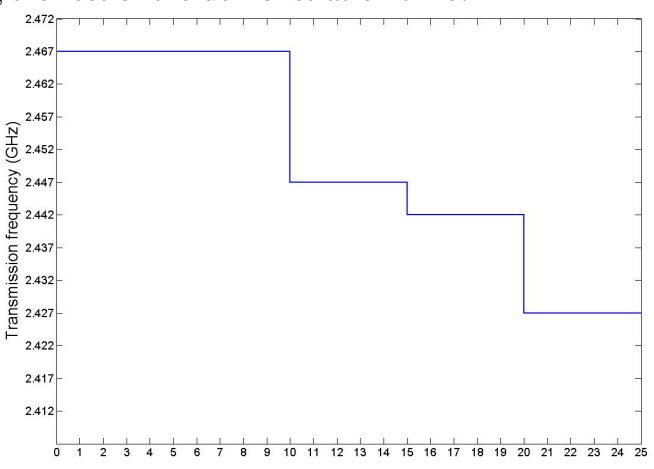

Figure 6. Transmission frequencies configured in USRP\#4

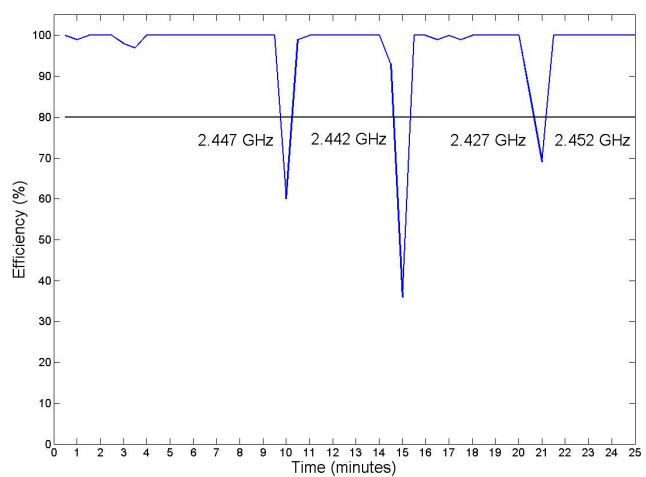

Figure 7. Efficiency in the data transmission through the ON. The frequencies assigned to the $\mathrm{ON}$ are indicated in the figure.

\section{Performance of the spectrum selection algorithm}

The purpose of this experiment is to evaluate the performance of the fittingness factor spectrum selection algorithm implemented in the testbed in relation to a random spectrum selection scheme. In this experiment, the communications' needs between the terminals in the ON are configured to generate sessions with a certain average duration and an inactivity time between them, in accordance to the configurations indicated in Table IV. At each session arrival, the ON is created. After creation, as shown in previous section, ON maintenance enables spectrum reselection by means of ONM procedures if needed.

For an easier illustration of the algorithm performance, and because of existing hardware limitations, this experiment considers just 2 spectrum blocks, centered at 2.472 and 2.484 $\mathrm{GHz}$, respectively, both with $5 \mathrm{MHz}$ bandwidth. USRP\#4 is configured to operate on the spectrum block centered at $2.472 \mathrm{GHz}$. The activity of this interference source is automatically adjusted following the transmission patterns indicated by the average activity/inactivity time durations in Table IV. No external interference is configured in the spectrum block centred at frequency $2.484 \mathrm{GHz}$ during the experiment. However, it is subject to some spurious uncontrolled interference existing in the environment. Statistics obtained in this spectrum block indicate that it is free of interference during $99.92 \%$ of the time.

TABLE IV. ACTIVITY PATTERNS OF THE ON AND THE INTERFERER USRP\#

\begin{tabular}{|c|c|c|c|c|}
\hline Experiment & $\begin{array}{c}\text { Average } \\
\text { inactivity } \\
\text { time ON }\end{array}$ & $\begin{array}{c}\text { Average } \\
\text { activity } \\
\text { time ON }\end{array}$ & $\begin{array}{c}\text { Average } \\
\text { inactivity } \\
\text { time } \\
\text { USRP\#4 }\end{array}$ & $\begin{array}{c}\text { Average } \\
\text { activity } \\
\text { time } \\
\text { USRP\#4 }\end{array}$ \\
\hline 1 & $30 \mathrm{~s}$ & $60 \mathrm{~s}$ & $300 \mathrm{~s}$ & $300 \mathrm{~s}$ \\
\hline 2 & $30 \mathrm{~s}$ & $60 \mathrm{~s}$ & $300 \mathrm{~s}$ & $60 \mathrm{~s}$ \\
\hline
\end{tabular}

Results compare the performance of the fittingness factor-based spectrum selection algorithm [14], in which the selection is done in accordance with statistics previously generated and stored about the fittingness factor behaviour in the existing spectrum blocks, against the random spectrum selection. In the later, the selection is done randomly among the available spectrum blocks. The duration of each experiment is 10 minutes. Whenever the measured efficiency in the $\mathrm{ON}$ falls below $80 \%$, the $\mathrm{ON}$ maintenance will trigger the ONM procedure to assign a different spectrum block. The results corresponding to the configuration of experiment 1 in Table IV are plot in Fig. 8 and Fig. 9 in terms of the efficiency observed by the ON with the random selection and the fittingness factor spectrum selection, respectively.

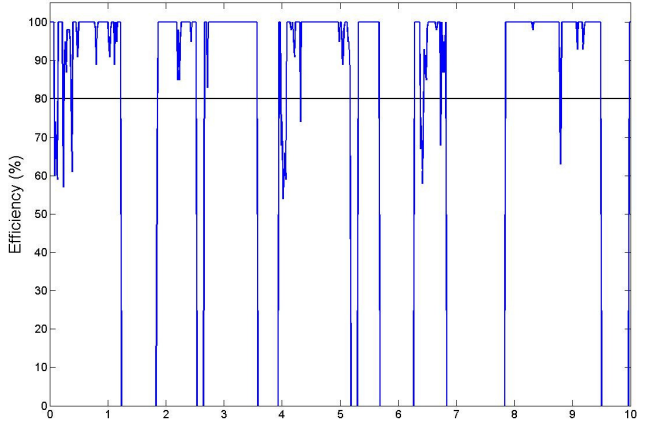

Figure 8. Efficiency in experiment 1 for the random selection algorithm

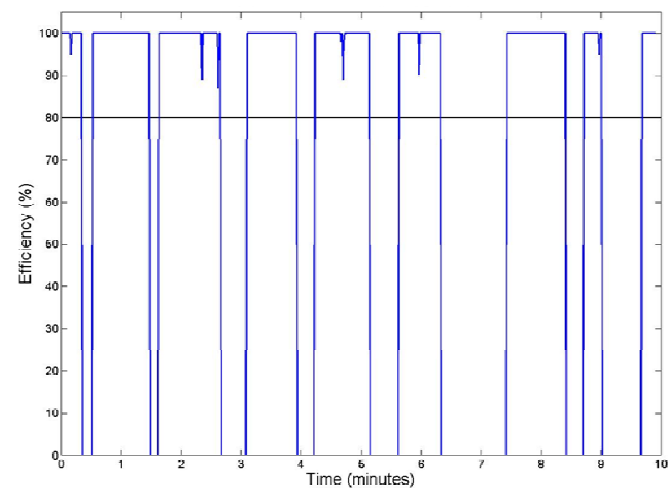

Figure 9. Efficiency in experiment 1 for the Fittingness factor-based spectrum selection algorithm 
Note that only the periods in which a session in the ON has been established are plot. Moreover, although the statistical pattern is the same for the two executions associated to the two algorithms, the actual durations of each session are different due to the randomness in the session generation. Table $\mathrm{V}$ presents the performance of the two algorithms in terms of the rate of executed ONM procedures, required to change the spectrum block allocated to the $\mathrm{ON}$ whenever there is degradation in the measured efficiency. This is a relevant metric in the comparison between spectrum selection algorithms since it reflects the associated signaling requirements. In experiment \#1, USRP\#4 is active $50 \%$ of the time, with an average duration much longer than the session duration of the ON (see Table IV). Correspondingly, in the random spectrum selection (see Fig. 8) ONM procedures need to be carried out frequently whenever the assigned spectrum block is the one used by USRP\#4. This can be observed in the figure because the efficiency falls below the limit of $80 \%$. The resulting ONM rate observed during the whole execution for experiment with the random algorithm is 2.2 procedures/min. On the contrary, with the fittingness factor based spectrum selection algorithm the efficiency is kept at a high level during the whole execution (see Fig. 9) and correspondingly no ONM procedures are required. In the configuration of experiment \#2, the duration of the interferer activity is much lower $(16 \%$ of the time with an average duration of 60s). As a result the ONM rate of the random selection is more reduced than with experiment 1 (i.e. 0.5 modifications $/ \mathrm{min}$ ), but still the fittingness factor spectrum selection achieves a better performance.

TABLE V. RATE OF ONM PROCEDURES (PROCEDURES/MIN)

\begin{tabular}{|c|c|c|}
\hline Experiment & Random selection & $\begin{array}{c}\text { Fittingness factor } \\
\text { spectrum selection }\end{array}$ \\
\hline 1 & 2.2 & 0.0 \\
\hline 2 & 0.5 & 0.0 \\
\hline
\end{tabular}

\section{VI.CONCLUSIONS AND NEXT STEPS}

In this paper, a testbed platform has been proposed to validate the spectrum opportunity identification and spectrum selection functionalities in $\mathrm{ON}$ management. It is based on reconfigurable devices able to transmit and receive at different operating frequencies, and implements the necessary signaling to support $\mathrm{ON}$ operation. Some results have been presented to validate the implementation conducted at the laboratory, to illustrate the reconfigurability capabilities of the ON links under varying interference conditions, and to evaluate experimentally the performance of the spectrum selection algorithm. The developed platform constitutes a powerful tool to support the development, assessment and validation of different algorithms in real operational radio environments. Aspects related to the practicality of the algorithmic solutions can be better assessed in the testbed rather than in a simulation environment. Robustness of the proposed solutions to unpredictable radio context conditions can also be proved in the platform. In this respect, intensive and extensive further evaluations and refinements on algorithmic solutions are expected in the near future.

\section{ACKNOWLEDGMENT}

This work has been supported by the Spanish Research Council and FEDER funds under ARCO grant (ref. TEC2010-15198).

\section{REFERENCES}

[1] V. Stavroulaki, et al. "Opportunistic Networks: An Approach for Exploiting Cognitive Radio Networking Technologies in the Future Internet", IEEE Vehicular Technology Magazine, Vol. 6, No. 3, pp. 52-59, September, 2011.

[2] O. Moreno (editor) "Business scenarios, technical challenges and system requirements", Deliverable D2.1 of OneFIT ICT project, October, 2010, available at http://www.ict-onefit.eu/.

[3] L. Catalin, K. V. Rama, A. Onur, B. Dusan, and S. Ivan, "Evaluation of energy-based spectrum sensing algorithm for vehicular networks" in Proceedings of the Software Defined Radio and Dynamic Spectrum Access Technical Conference, Washington, DC, December, 2009.

[4] M. A. Sarijari, A. Marwanto, N. Fisal, S. K. S. Yusof, R. A. Rashid, and M. H. Satria, "Energy Detection Sensing based on GNU Radio and USRP: An Analysis Study"; Malaysia Int. Conf. on Comm. (MICC), Kuala Lumpur, December, 2009.

[5] R. Miller, W. Xu, P. Kamat, and W. Trappe: "Service Discovery and Device Identification in Cognitive Radio Networks", SDRN conference, London, December, 2007.

[6] Q. Zhao and A. Swami, "A Decision-Theoretic Framework for Opportunistic Spectrum Access", IEEE Wireless Communications, Vol. 14, No. 4, August, 2007.

[7] H. Li, G. Zhu, Z. Liang, and Y. Chen, "A Survey on Distributed Opportunity Spectrum Access in Cognitive Network", WiCOM, Chengdu, September 2010.

[8] Q. Zhao, S. Geirhofer, L. Tong, and B.M. Sadler, "Optimal Dynamic Spectrum Access via Periodic Channel Sensing", Wireless Communications and Networking Conference (WCNC) Hong Kong, March 2007.

[9] S.D. Jones, N. Merheb, and I.J. Wang, "An Experiment for Sensing-Based Opportunistic Spectrum Access in CSMA/CA Networks", DySPAN, Baltimore, November 2005.

[10] L. Ma, X. Han, and C.C. Shen, "Dynamic Open Spectrum Sharing MAC Protocol for Wireless Ad Hoc Networks", DySPAN, Baltimore, November 2005.

[11] ETSI, TC RRS, "Reconfigurable Radio Systems; Functional Architecture for the Management and the Control of Reconfigurable Radio Systems", TR 102 682, July 2009.

[12] J. Gebert (editor) "OneFIT functional and system architecture", Deliverable D2.2 of OneFIT project, February, 2011, available at http://www.ict-onefit.eu/.

[13] Robin I.C. Chiang, Gerard B. Rowe, and Kevin W. Sowerby, "A Quantitative Analysis of Spectral Occupancy Measurements for Cognitive Radio", VTC Spring, Dublin, April, 2007.

[14] F. Bouali, O. Sallent, J. Pérez-Romero, and R. Agustí, "Exploiting Knowledge Management for Supporting Spectrum Selection in Cognitive Radio Networks", CROWNCOM conference, Stockholm, June, 2012.

[15] http:// www.ettus. com.

[16] http://www.gnuradio.org.

[17] ETSI TR 102684 "RRS; Feasibility Study on Control Channels for Cognitive Radio Systems", April, 2012.

[18] T. Wierzbowski (editor) "Protocols, performance assessment and consolidation on interfaces for standardization", Deliverable D3.3 of OneFIT ICT project, June, 2012. 